\title{
THE TAYLOR ESTIMATE OF RECOVERABLE STRAINS IN SHAPE-MEMORY POLYCRYSTALS
}

\author{
K. BHATTACHARYA*, R.V. KOHN** and Y.C. SHU* \\ * California Institute of Technology, Pasadena, CA 91125, USA. \\ ** Courant Institute of Mathematical Sciences, \\ 251 Mercer Street, New York, NY 10012, USA.
}

\section{Introduction}

Shape-memory behavior is the ability of certain materials to recover, on heating, apparently plastic deformation sustained below a critical temperature. Some materials have good shape-memory behavior as single crystals but little or none as polycrystals, while others have good shape-memory behavior even as polycrystals. Bhattacharya and Kohn $(1996,1997)$ have proposed a framework to understand this difference. They use energy minimization and the Taylor estimate to argue that the recoverable strains in a polycrystal depend not only on the texture of the polycrystal and the transformation, but critically on the change in symmetry during the underlying martensitic phase transformation. Their results agree with the experimental observations. Shu and Bhattacharya (1997) have also used the Taylor estimate to study the effect of texture in polycrystals of Nickel-Titanium and Copper based shape-memory alloys. The use of the Taylor estimate was evaluated in some detail in Bhattacharya and Kohn (1997) and more recently in Shu and Bhattacharya (1997) and Shu (1997). In this short report, we summarize the model of recoverable strain and discuss some results that allow us to evaluate the Taylor estimate.

Saburi and Nenno (1981) have given a very insightful discussion about recoverable strains. Our work can be seen as an attempt to make their ideas more quantitative. The source of the shape-memory effect is a martensitic phase transformation. A material which undergoes a martensitic phase transformation has two distinct crystalline structures: the parent or austenite phase, preferred at high temperatures; and the product or martensite phase, preferred at low temperatures. Typically, the austenite has greater symmetry than the martensite; consequently, the martensite phase occurs in $k$ different symmetry-related variants. The variants have the same crystalline structure, but have a different orientation relative to the austenite lattice. The number of variants $k$ depends on the change of symmetry. There are 3 variants for a cubic-tetragonal transformation, 4 for cubic-trigonal, 6 for cubic-orthorhombic, and 12 for cubic-monoclinic. 
Consider a single crystal of the high temperature austenite phase. As it is cooled below the transformation temperature, the transformation takes place, creating a mixture of variants of martensite. While the transformation strain of each variant is different, the macroscopic effect of the transformation is negligible due to self-accommodation between the different variants. Now deform the sample. The variants rearrange themselves, if they can, so as to remain essentially stress-free by changing the microstructure. The resulting deformation appears macroscopically plastic: there is no restoring force since the variants in their new configuration are not stressed. But in fact, it is recoverable: heating the crystal above its transformation temperature turns each variant of martensite back to austenite and the crystal springs back to its original shape. Notice, however, that only certain strains can be recovered: those that can be achieved by the rearrangement of martensite variants. Larger strains will introduce stress, leading to lattice defects and nonrecoverability. Therefore, the recoverable strains for a single crystal can be identified with the strains achievable by stress-free mixtures of martensite variants.

Now consider a polycrystal, consisting (in the austenite phase) of a large number of grains with different orientations. On cooling, each grain transforms to a self-accommodated mixture of martensite variants. As the polycrystal is deformed, each grain tries to accommodate the strain by adjusting its microstructure of stress-free variants. The deformation is recoverable if they succeed. Therefore, the recoverable strains for a polycrystal are the macroscopic averages of locally varying strain fields which can be accommodated within each grain by rearrangement of the martensite variants.

We wish to calculate the set of recoverable strains in a polycrystal. Energy minimization provides a very convenient framework to formulate the ideas stated above.

\section{Energy Minimization and Recoverable Strain}

\subsection{THE RECOVERABLE STRAIN OF A SINGLE CRYSTAL}

Consider a single crystal of the austenite. Choose this as the reference configuration. Therefore, the austenite has stress-free strain $e^{(o)}=0$. The stress-free strain of the $i$ th variant of martensite, $e^{(i)}$, can be calculated from the lattice parameters. We assume that given some temperature and boundary condition the single crystal will occupy a state that minimizes the total energy

$$
\int_{\Omega} \varphi(e(u)) d x
$$

Here $\Omega$ is the region occupied by the crystal; $e(u)=\frac{1}{2}\left(\nabla u+\nabla u^{T}\right)$ is the linear strain associated with displacement $u$. We shall refer to the integrand $\varphi$ as the microscopic elastic energy. It depends on temperature. We are interested in temperatures below the transformation temperature. Here, the microscopic energy is 
nonconvex with multiwell structure - one well for each variant. For our purposes, it suffices to assume that the microscopic elastic energy is given by:

$$
\varphi(e)=\min _{i=1, \ldots, k}\left\{\frac{1}{2}\left|e-e^{(i)}\right|^{2}\right\} .
$$

Notice that we have assumed that each variant is elastic. As a matter of convenience, we have assumed that the elastic modulus is identical (and equal to identity) in each of the variants. We have also ignored the austenite well (since it is higher than the martensite wells at this temperature and we are going to minimize the energy).

Energy minimization with this multiple well energy leads to minimizing sequences which we interpret as microstructure or fine-scale mixtures of the different variants of martensite (Ball and James 1987). Therefore, the behavior of a single crystal is governed not by the microscopic energy, $\varphi$, but the mesoscopic energy $\hat{\varphi}$. Physically, $\hat{\varphi}(\boldsymbol{e})$ is the average stored energy when the average strain is $e$ after taking into account the microstructure. Mathematically, it is obtained by the relaxation of $\varphi$ and may be defined as

$$
\hat{\varphi}(e)=\min _{u=e \cdot x \text { at } \partial \Omega} \frac{1}{\operatorname{Vol} . \Omega} \int_{\Omega} \varphi(e(u)) d x .
$$

We note that the definition (3) does not really depend on the choice of domain $\Omega$. There are equivalent definitions using periodic rather than affine boundary conditions, or Young measures rather than spatial averages. Also note the elementary but important fact that the minimum value of $\hat{\varphi}$ is the same as that of $\varphi$.

We can now give a mathematically precise formulation of recoverable strains. The recoverable strains for a single crystal are the minimizers of its mesoscopic energy. Therefore, we can define the set of recoverable strains for a single crystal,

$$
\mathcal{S}=\{e: \hat{\varphi}(e)=0\} .
$$

In general, it is very difficult to evaluate the set $\mathcal{S}$ (see for example, Bhattacharya et al 1994). However, the following result from Bhattacharya (1993) allows us to evaluate it in physically relevant cases including cubic-tetragonal, cubic-trigonal and cubic-orthorhombic transformations.

Proposition 1 Let us call two linear strains $f$ and $g$ "elastically compatible" if they satisfy $f-g=\frac{1}{2}(a \otimes n+n \otimes a)$ for some vectors $a$ and $n$. Suppose the stressfree strains $\boldsymbol{e}^{(1)}, \ldots, e^{(k)}$ are pairwise elastically compatible. Then the associated set $\mathcal{S}$, defined by (4), is simply their convex hull.

The cubic-monoclinic case is different: its stress-free strains are not pairwise compatible, and the associated set $\mathcal{S}$ is not the convex hull. This complicates the analysis, but we can study this case by embedding it into the cubic-orthorhombic case (Bhattacharya and Kohn 1996).

We can already begin to see that the number of variants is crucially important. Note that the convexification of $\varphi$ is always a lower bound for $\hat{\varphi}$. Therefore $\mathcal{S}$ is 
always contained in the convex hull of $\left\{e^{(i)}\right\}_{i=1}^{k}$. Since the martensite variants are symmetry related, they all have the same trace, i.e. $\operatorname{tr} e^{(1)}=\ldots=\operatorname{tr} e^{(k)}$. Thus $\mathcal{S}$ lies in a $(k-1)$-dimensional subset of the 5-dimensional "deviatoric hyperplane" $\operatorname{tr} e=\operatorname{tr} e^{(i)}$. When $k<6$, e.g. when the phase transformation is cubic-tetragonal or cubic-trigonal, $\mathcal{S}$ is dimensionally deficient: its dimension is strictly smaller than that of the deviatoric hyperplane. When $k \geq 6$, on the other hand, counting suggests that $\mathcal{S}$ should be a set with interior in the deviatoric hyperplane.

Another important fact is that when the austenite is cubic and the martensite is tetragonal, trigonal, orthorhombic, or monoclinic, $\mathcal{S}$ contains the isotropic strain

$$
\mathcal{I}=\frac{1}{3} \operatorname{tr} e^{(i)} I
$$

This follows from the characterizations of $\mathcal{S}$ given in Bhattacharya and Kohn (1996). It is linked to the phenomenon of self-accommodation (Bhattacharya 1992).

\subsection{THE RECOVERABLE STRAIN OF A POLYCRYSTAL}

A polycrystal is an assemblage of grains, each composed of the same shape-memory material in a different orientation. We describe the texture of a polycrystal by a rotation-valued function $R(x) . R(x)$ gives the orientation of the crystal at the point $x$ relative to some fixed reference crystal. In a typical polycrystal, $R$ is piecewise constant, though we shall not assume any such restriction in what follows.

The total elastic energy stored in a polycrystal is given by

$$
\int_{\Omega} \varphi\left(R^{T}(x) e(u) R(x)\right) d x
$$

in place of (1). However, we know that energy minimization leads to minimizing sequences and microstructure. Therefore, the minimization problem does not change if we replace the microscopic energy $\varphi$ in (6) with the mesoscopic energy $\hat{\varphi}$ (Acerbi and Fusco 1984). Physically, we are assuming that the length scale of the martensitic microstructure is smaller than that of the grains.

In order to discuss the behavior of the polycrystal, we introduce the macroscopic energy of the polycrystal $\bar{\varphi} . \bar{\varphi}$ is obtained from $\hat{\varphi}$ by homogenization. A convenient definition is based on affine boundary conditions:

$$
\bar{\varphi}(\bar{e})=\min _{u=\bar{e} \cdot x \text { at } \partial \Omega} f_{\Omega} \hat{\varphi}\left(R^{T}(x) e(u) R(x)\right) d x .
$$

This has the advantage of being easy to use, however the resulting $\bar{\varphi}$ depends on the details of the domain $\Omega$ and the texture $R(x)$. One might say that it describes a "specific sample" of polycrystal. One can provide alternate definitions based on periodic polycrystals, random polycrystals or Gamma convergence. We do not fuss about which distinctions between these; instead we use the definition which is most convenient for the purposes at hand. A more careful reader is referred to Bhattacharya and Kohn (1997). 
We are now in a position to give a mathematically precise meaning for recoverable strains in a polycrystal. The recoverable strains for a polycrystal are the minimizers of its macroscopic energy. Therefore, we define the set of recoverable strains for a polycrystal

$$
\mathcal{P}=\{e: \bar{\varphi}(e)=0\} .
$$

Notice that we have implicitly assumed that $\bar{\varphi}$ has minimum value 0 . This is a consequence of (5). The isotropic tensor $\mathcal{I}$ is in $\mathcal{S}$, so $\hat{\varphi}\left(R^{T}(x) \mathcal{I} R(x)\right)=\hat{\varphi}(\mathcal{I})=0$ for every $x$. It follows that $\bar{\varphi}(\mathcal{I})=0$.

\section{The Taylor Estimate and its Implications}

Our task is now clear. To assess the recoverable strain of a shape-memory polycrystal we must consider the associated macroscopic energy $\bar{\varphi}$, and estimate the set $\mathcal{P}$ where $\vec{\varphi}=0$. This task might at first appear hopeless. However, there is an elementary but fundamental bound, based on the use of a constant-strain test field:

Proposition 2 The set $\mathcal{P}$ of recoverable strains contains at least the set

$$
\mathcal{T}=\bigcap_{x \in \Omega} \mathcal{S}(x)=\left\{e: R^{T}(x) e R(x) \in \mathcal{S} \text { for each } x \in \Omega\right\}
$$

We call this the "Taylor bound" to highlight the analogy with Taylor's uniformstrain hypothesis from polycrystal plasticity (Taylor 1938 and Bishop and Hill 1951). The physical meaning of the Taylor bound is clear. It describes the strains which can be accommodated without making use of cooperative effects between grains.

Proposition 2 says only that $\mathcal{P} \supset \mathcal{T}$. It is silent concerning how much bigger $\mathcal{P}$ might be. We believe, however, that $\mathcal{T}$ is usually a good indicator for $\mathcal{P}$. More precisely, we conjecture that for polycrystals with sufficient symmetry (and under appropriate hypothesis on the set $\mathcal{S}$ ), $\mathcal{P}$ has the same dimension as $\mathcal{T}$. We therefore like to call $\mathcal{T}$ the Taylor estimate.

For materials of interest, the set $\mathcal{T}$ is never empty: it always contains at least the strain $\mathcal{I}$, by (5). We say the Taylor bound is trivial if $\mathcal{T}$ consists of just this one point. For such polycrystals Proposition 2 says only that $\mathcal{I} \in \mathcal{P}$, but our conjecture says that $\mathcal{P}=\{\mathcal{I}\}$. In other words, such polycrystals should have no recoverable strain.

Let us explain heuristically why $\mathcal{P}$ should be trivial if $\mathcal{T}$ is. We start with the observation that for $\mathcal{T}$ to be trivial, $\mathcal{S}$ must be dimensionally deficient, i.e. it must not span the entire deviatoric strain space. So the constraint $e(x) \in \mathcal{S}(x)$ restricts the mesoscopic strain to lie in a lower dimensional set, varying from grain to grain. This is a severe algebraic restriction. We expect it to be inconsistent with the linear differential constraints $e_{i j, k l}+e_{k l, i j}=e_{i k, j l}+e_{j l, i k}$ which must be satisfied if $\mathrm{e}(x)$ is to come from a deformation. 
Our conjecture provides the essential link between the symmetry of the phase transition and the recoverable strains of polycrystals. When there are fewer than 6 variants (tetragonal or trigonal martensite) the set $\mathcal{S}$ is dimensionally deficient, and $\mathcal{T}$ is trivial. We conjecture that $\mathcal{P}$ is also trivial. Therefore such materials lose their shape-memory behavior when formed into unstructured polycrystals. When there are 6 or more variants (orthorhombic or monoclinic martensite) the set $\mathcal{S}$ contains a neighborhood of $\mathcal{I}$ in the deviatoric hyperplane tr $e=$ constant, so the same is true of $\mathcal{T}$ and $\mathcal{P}$. Polycrystals made from such materials can recover some strain in any deviatoric direction. The maximum recoverable strain is unclear, but we expect it to exceed the conservative estimate $\mathcal{T}$.

\section{Model Problems in Two Dimensions}

In this section, we consider two model problems, the $2 D$ diagonal, trace-free elastic material and the $2 D$ diagonal elastic material. For polycrystals made from first material we prove that $\mathcal{P}$ has the same dimension as $\mathcal{T}$. For polycrystals made from the second we show that the dimension of $\mathcal{P}$ can be larger than that of $\mathcal{T}$. This demonstrates that the accuracy of the Taylor estimate is a problem-specific matter rather than a universal one.

\subsection{D DIAGONAL, TRACE-FREE ELASTIC MATERIAL}

In this case the microscopic energy is given by (2) with $k=2$ and

$$
e^{(1)}=\left(\begin{array}{rr}
1 & 0 \\
0 & -1
\end{array}\right) \quad e^{(2)}=\left(\begin{array}{rr}
-1 & 0 \\
0 & 1
\end{array}\right) \text {. }
$$

It is possible to show that the mesoscopic energy is

$$
\hat{\varphi}\left(\begin{array}{cc}
a+b & c \\
c & a-b
\end{array}\right)=\frac{1}{2}\left[a^{2}+(|b|-1)_{+}^{2}+c^{2}\right] .
$$

It follows that $\mathcal{S}$ is the line segment from $e^{(1)}$ to $e^{(2)}$,

$$
\mathcal{S}=\left\{\left(\begin{array}{cc}
b & 0 \\
0 & -b
\end{array}\right):|b| \leq 1\right\},
$$

and the Taylor estimate is trivial:

$$
\mathcal{T}=\{0\}
$$

We can prove the following theorem for this material (Bhattacharya and Kohn 1997).

Theorem 3 For any polycrystal made from the 2D diagonal, trace-free material, the macroscopic energy satisfies

$$
\bar{\varphi}(e)+\bar{\varphi}\left(R_{\pi / 4}^{T} e R_{\pi / 4}\right) \geq c_{1}(\operatorname{tr} e)^{2}+c_{2}\left|e^{D}\right|^{4} .
$$


Here $c_{1}$ and $c_{2}$ are texture-independent constants, and the estimate is only asserted when $e^{D}=e-\frac{1}{2}(\operatorname{tr} e) I$ is sufficiently small.

This theorem tells us that for any polycrystal sufficientsymmetry, $\mathcal{P}=\{0\}$. And thus this result is consistent with our conjecture that the dimension of $\mathcal{T}$ agrees with the dimension of $\mathcal{P}$. This theorem can be proved using the translation method with the translation that has been used by Lurie and Cherkaev (1984) as well as Avellaneda et al. (1996) to bound the effective shear modulus of a 2D linear elastic polycrystal. However the argument has a small twist. There, the analog of the mesoscopic energy is quadratic at the origin and one simply needs to use the non-negativity of the translated integrand. Here, the mesoscopic energy is not quadratic at the origin and hence one needs to use the boundedness of the Fenchel transform of the translated integrand. This is what gives the rather unexpected exponent of 4 in the lower bound (14).

We wondered whether the exponent is an artifact of the method. We have not completely resolved this; however we will now argue that the behavior of a polycrystal with sufficient symmetry is subquadratic near the origin. The essence of the matter is that linearization and homogenization should commute (Geymonat et al 1993). To explain what this means, we note that the quadratic approximation of the mesoscopic energy near 0 is

$$
\hat{\varphi}_{\text {lin }}\left(\begin{array}{cc}
a+b & c \\
c & a-b
\end{array}\right)=\frac{1}{2}\left[a^{2}+c^{2}\right] .
$$

Hence this linear material is degenerate in the sense that the shear moduli are 0 and 1. "Linearization then homogenization" means starting with the linear polycrystal whose local energy is $\hat{\varphi}_{\text {lin }}\left(R^{T}(x) e R(x)\right)$, then passing in the usual way to its effective energy $\bar{\varphi}_{\text {lin }}(e)$. "Homogenization then linearization" means considering our nonlinear macroscopic energy $\bar{\varphi}$, then taking its quadratic approximation near $e=0$. If the two operations commute then we should have

$$
\bar{\varphi}(e)=\bar{\varphi}_{\mathrm{lin}}(e)+o\left(|e|^{2}\right) .
$$

We can use the results by Lurie and Cherkaev (1984) for any polycrystal with sufficientsymmetry, $\bar{\varphi}_{\text {lin }}(e)=(\text { tre })^{2}+\mathrm{c}\left|\mathrm{e}^{\mathrm{D}}\right|^{2}$ with $c=0$. So we conclude that $\bar{\varphi}$ is flatter than quadratic. The general statement that linearization and homogenization commute requires strict convexity of the energy $\hat{\varphi}$; however, we can adapt the proof to this problem (Bhattacharya and Kohn 1997).

\subsection{D DIAGONAL ELASTIC MATERIAL}

In this case the microscopic energy is given by (2) with $k=4$ and

$$
e^{(1)}=\left(\begin{array}{ll}
1 & 0 \\
0 & 1
\end{array}\right) e^{(2)}=\left(\begin{array}{rr}
1 & 0 \\
0 & -1
\end{array}\right) e^{(3)}=\left(\begin{array}{rr}
-1 & 0 \\
0 & 1
\end{array}\right) e^{(4)}=\left(\begin{array}{rr}
-1 & 0 \\
0 & -1
\end{array}\right) \text {. }
$$


The mesoscopic energy is

$$
\hat{\varphi}(e)=\frac{1}{2}\left[\left(\left|e_{11}\right|-1\right)_{+}^{2}+\left(\left|e_{22}\right|-1\right)_{+}^{2}+2 e_{12}^{2}\right] .
$$

The set of recoverable strains for the single crystal is the convex hull of $\left\{e^{(i)}\right\}$, a two-dimensional square in the three-dimensional space of symmetric matrices:

$$
\mathcal{S}=\left\{e:\left|e_{11}\right| \leq 1,\left|e_{22}\right| \leq 1, e_{12}=0\right\} .
$$

This is a consequence of Proposition 1, applied separately to $\left\{e^{(1)}, e^{(2)}, e^{(3)}\right\}$ and to $\left\{e^{(2)}, e^{(3)}, e^{(4)}\right\}$. The Taylor estimate contains only multiples of the identity:

$$
\mathcal{T}=\bigcap_{R \in S O(2)} R S R^{T}=\{a I:|a| \leq 1\} ;
$$

in particular, it is one-dimensional.

This example is interesting because the Taylor estimate is not reliable in this case, not even for polycrystals which are macroscopically isotropic. Consider the polycrystal obtained by layering the basic crystal with its rotation by $\pi / 4$ using layers normal to $(1,1)$ and equal volume fraction. It is very easy to show that $\bar{\varphi} \equiv 0$ in a neighborhood of 0 for this laminated polycrystal. Though this rankone laminate is highly anisotropic we can use it to make isotropic polycrystals by mixing it with itself in different orientations. Thus, one can get isotropic polycrystals of the material with $\bar{\varphi} \equiv 0$ in a neighborhood of 0 . Thus $\mathcal{P}$ can be three-dimensional even though $\mathcal{T}$ is one-dimensional.

\section{Cubic-Tetragonal Material}

Consider a material that undergoes cubic to tetragonal transformation. It has $k=3$ variants and

$$
e^{(1)}=\left(\begin{array}{ccc}
\alpha & 0 & 0 \\
0 & \alpha & 0 \\
0 & 0 & \beta
\end{array}\right), e^{(2)}=\left(\begin{array}{ccc}
\alpha & 0 & 0 \\
0 & \beta & 0 \\
0 & 0 & \alpha
\end{array}\right), e^{(3)}=\left(\begin{array}{ccc}
\beta & 0 & 0 \\
0 & \alpha & 0 \\
0 & 0 & \alpha
\end{array}\right) .
$$

It is then a matter of calculation to show (Bhattacharya and Kohn 1996) that

$$
\mathcal{S}=\left\{e \| \begin{array}{l}
e_{12}=e_{13}=e_{23}=0 \\
e_{11}+e_{22}+e_{33}=2 \alpha+\beta \& \\
\min \{\alpha, \beta\} \leq e_{11}, e_{22}, e_{33} \leq \max \{\alpha, \beta\}
\end{array}\right\}
$$

There is no loss of generality if we restrict ourself to the volume-preserving case $\beta=-2 \alpha$. We may also suppose $\alpha>0$.Then $\mathcal{S}$ is an equilateral triangle centered at the origin in the two-dimensional subspace $V$ of diagonal, trace-free strains,

$$
V=\left\{\left(\begin{array}{ccc}
a & 0 & 0 \\
0 & b & 0 \\
0 & 0 & c
\end{array}\right): a+b+c=0\right\} .
$$


The vertices of $\mathcal{S}$ are $\operatorname{diag}[\alpha, \alpha,-2 \alpha], \operatorname{diag}[\alpha,-2 \alpha, \alpha]$, and $\operatorname{diag}[-2 \alpha, \alpha, \alpha]$.

\subsection{UNIAXIAL POLYCRYSTAL}

We now consider polycrystals with $\langle 001\rangle$ texture, in other words those in which all the rotations leave the $x_{3}$-axis unchanged. No restriction is placed on the geometry of the grains.

It is not convenient to work with the convexification of $\varphi$ this time. Instead we shall use the more symmetric function

$$
\hat{\varphi}(e)=\frac{1}{2}\left[\left(\left|e^{\prime}\right|-\rho\right)_{+}^{2}+\left|e^{\prime \prime}\right|^{2}\right]
$$

where $e^{t}$ and $e^{\prime \prime}$ are the orthogonal projections of $e$ onto $V$ and $V^{\perp}$ :

$$
e=\pi_{V} e+\left(I-\pi_{V}\right) e=e^{\prime}+e^{\prime \prime},
$$

and $\rho=\sqrt{6} \alpha$ is the radius of the smallest ball in $V$ containing $\mathcal{S}$. This $\hat{\varphi}$ is a convex lower bound for the relaxation of (2).

The sets $\mathcal{S}, \mathcal{T}$, and $\mathcal{P}$ associated with the real cubic-tetragonal energy are different from those associated with (24). To avoid confusion we shall write $\mathcal{S}_{\text {symm }}$, $\mathcal{T}_{\text {symm }}, \mathcal{P}_{\text {symm }}$ for the sets defined using the more symmetric energy (24). Since this is a lower bound for the true cubic-tetragonal energy we have

$$
\mathcal{S} \subset \mathcal{S}_{\text {symm }}, \quad \mathcal{T} \subset \mathcal{T}_{\text {symm }}, \quad \mathcal{P} \subset \mathcal{P}_{\text {symm }} .
$$

We have evaluated the Taylor estimate in Bhattacharya and Kohn (1996) for a polycrystal with this texture. When specialized to the incompressible case that calculation gives

$$
\mathcal{T}=\left\{\left(\begin{array}{rrr}
a & 0 & 0 \\
0 & a & 0 \\
0 & 0 & -2 a
\end{array}\right):-\alpha / 2 \leq a \leq \alpha\right\},
$$

and a similar calculation gives

$$
\mathcal{T}_{\text {symm }}=\left\{\left(\begin{array}{rrr}
a & 0 & 0 \\
0 & a & 0 \\
0 & 0 & -2 a
\end{array}\right):|a| \leq \alpha\right\} .
$$

In this case, we can prove the following theorem (Bhattacharya and Kohn 1997) using the translation method.

Theorem 4 For the symmetrized cubic-tetragonal energy (24), any uniaxial polycrystal satisfies

$$
\bar{\varphi}(e)+\bar{\varphi}\left(R_{\pi / 4}^{T} e R_{\pi / 4}\right) \geq c_{1}\left[(\operatorname{tr} e)^{2}+e_{13}^{2}+e_{23}^{2}\right]+c_{2} \rho^{-2}\left[\left(e_{11}-e_{22}\right)^{2}+4 e_{12}^{2}\right]^{2} .
$$

Here $c_{1}$ and $c_{2}$ are absolute constants, independent of texture and the value of $\rho$. The estimate is asserted only when $\left(e_{11}-e_{22}\right)^{2}+4 e_{12}^{2}$ is sufficiently small. 


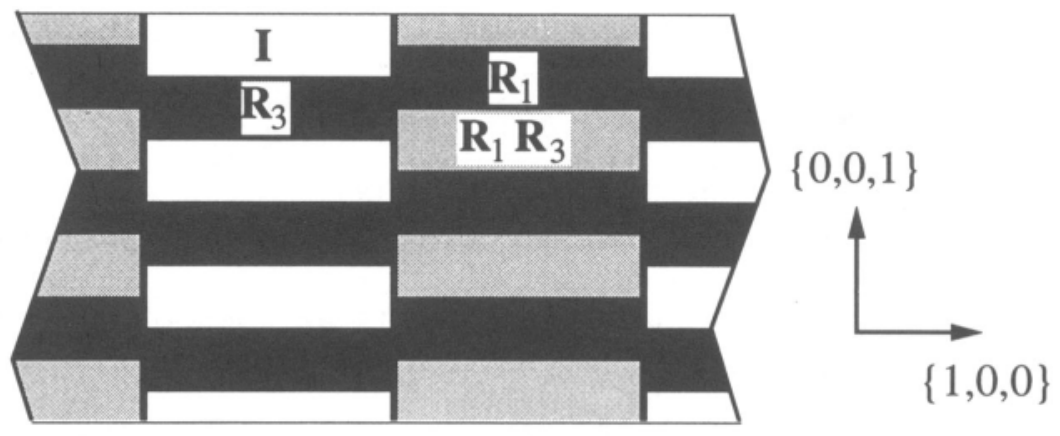

Figure 1: A rank-two laminated polycrystal. $R_{1}$ is a rotation about the axis $\{1,0,0\}$ while $R_{3}$ about the axis $\{0,0,1\}$. We can pick the angles of these rotations arbitrarily as long as they do not take the values $n \pi / 2$ for $n$ integer.

This theorem tells us that for any uniaxial polycrystal with sufficient transverse symmetry, the dimension of $\mathcal{P}_{\text {symm }}$ is at most one. Therefore, according to (26), the dimension of $\mathcal{P}$ is also at most one. This agrees with the dimension of $\mathcal{T}$ and hence this result is consistent with our conjecture.

\subsection{GENERAL POLYCRYSTAL}

We now consider polycrystals which do not have a $\langle 001\rangle$ texture. In this case, the Taylor estimate $\mathcal{T}$ is trivial (Bhattacharya and Kohn 1996). We conjecture in a polycrystal with sufficient symmetry, the set $\mathcal{P}$ will also be trivial. Unfortunately, we have not been able to prove (or contradict) such a result. However, we present the following example in support of our conjecture.

Consider the rank-two laminated polycrystal shown in Figure 1. It is possible to show that there exists $C>0$ such that

$$
\bar{\varphi}(e) \geq C|e|^{2} .
$$

Therefore, for this polycrystal, the set $\mathcal{P}$ is trivial. Also note that we have a quadratic lower bound in contrast to the situations in the 2D diagonal, tracefree elastic material and the uniaxial polycrystal. At this time we are unable to comment on the reasons or the significance of this difference.

Laminated polycrystals have been studied in some detail in (Shu and Bhattacharya 1997 and Shu 1997). We also show that for any finite-rank laminated polycrystal made of this material,

$$
\text { dimension } \mathcal{P} \leq 2 .
$$

In other words, the dimension of $\mathcal{P}$ can be no greater than the dimension of $\mathcal{S}$. 
Further, for any such polycrystal with sufficient symmetry, the dimension of $\mathcal{P}$ is indeed zero and hence equal to the dimension of $\mathcal{T}$.

\section{Discussion}

We have conjectured that for polycrystals with sufficient symmetry, $\mathcal{P}$ has the same dimension as $\mathcal{T}$. Here is a summary of the results in its favor.

- Bhattacharya and Kohn (1997) give a very detailed analysis of a scalar model problem where the Taylor estimate is trivial and for any polycrystal with sufficient symmetry $\mathcal{P}$ is also trivial.

- We have discussed a similar example in Section 4.1 in two-dimensional elasticity.

- The uniaxial polycrystal made from the cubic-tetragonal material has a onedimensional Taylor estimate (Section 5.1). We have shown that for polycrystals with sufficient (transverse) symmetry $\mathcal{P}$ is also one dimensional.

- We construct an example of a laminated polycrystal of a material undergoing cubic-tetragonal transformation where the Taylor bound is trivial. We show that the set $\mathcal{P}$ is also trivial (Section 5.2). The behavior of laminated polycrystals of a material undergoing cubic-trigonal transformation is also very similar.

There is also one result running contrary. For our 2D diagonal elastic material, the Taylor estimate is one-dimensional, however there are polycrystals, even isotropic ones, for which $\mathcal{P}$ is three-dimensional (Section 4.2). Thus our conjecture is false in this case. In particular, it cannot be taken as a universally valid assertion about nonlinear homogenization. Rather, it must reflect some (as yet undetermined) feature(s) of the set $\mathcal{S}$.

We wonder whether the following "lamination test" is one such feature. Let us denote by $\mathcal{P}_{\text {lam }}$ the set of recoverable strains for any rank-one laminated polycrystal. The lamination test would say that if $\operatorname{dim}\left(\mathcal{P}_{\text {lam }}\right) \leq \operatorname{dim}(\mathcal{S})$ for every rank-one laminated polycrystal, then the $\operatorname{dim}(\mathcal{P})=\operatorname{dim}(\mathcal{T})$ in a polycrystal with sufficient symmetry. In other words, our original conjecture will fail exactly when our set $\mathcal{S}$ is such that we can increase the set of recoverable sets by simple lamination. This is consistent with the 2D diagonal elastic material in Section 4.2. And this is in some sense the spirit behind the use of lamination in the cubic-tetragonal material in Section 5.2. However, we are far from proving such a result. Indeed, the fact that the class of rank-one convex functions is different from the class of quasiconvex functions (Šveràk 1992) says that one should be cautious about such a lamination test.

Acknolwedgment We gratefully acknowledge the partial financial support of the AFOSR, ARO and NSF. 


\section{References}

[1] Acerbi, E. and Fusco, N. (1984) Semicontinuity problems in the calculus of variations, Arch. Rat. Mech. Anal. 86, 125-145.

[2] Avellaneda, M., Cherkaev, A.V., Gibiansky, L.V., Milton, G.W., and Rudelson, M. (1996) A complete characterization of the possible bulk and shear moduli of planar polycrystals, J. Mech. Phys. Solids 44, 1179-1218.

[3] Ball, J.M. and James, R.D. (1987) Fine phase mixtures as minimizers of energy, Arch. Rat. Mech. Anal. 100, 13-52.

[4] Bhattacharya, K. (1992) Self-accommodation in martensite, Arch. Rat. Mech. Anal. 120, 201-244.

[5] Bhattacharya, K. (1993) Comparison of the geometrically nonlinear and linear theories of martensitic transformation, Cont. Mech. Thermodyn. 5, 205-242.

[6] Bhattacharya, K., Firoozye, N.B., James, R.D. and Kohn, R.V. (1994) Restrictions on microstructure, Proc. Roy. Soc. Edinburgh 124A, 843-878.

[7] Bhattacharya, K. and Kohn, R.V. (1996) Symmetry, texture, and the recoverable strain of shape-memory polycrystals, Acta Mater. 44, 529-542.

[8] Bhattacharya, K. and Kohn, R.V. (1997) Elastic energy minimization and recoverable strains of polycrystalline shape-memory materials, Arch. Rat. Mech. Anal. 139, 99-180.

[9] Bishop, J.F.W. and Hill, R. (1951) A theory of the plastic distortion of a polycrystalline aggregate under combined stresses, Phil. Mag. 42, 414-427.

[10] Geymonat, G., Müller, S., and Triantafyllidis, N. (1993) Homogenization of nonlinearly elastic materials, microscopic bifurcation and macroscopic loss of rank-one convexity, Arch. Rat. Mech. Anal. 122, 231-290.

[11] Lurie, K.A. and Cherkaev, A.V. (1984) G- closure of some particular sets of admissible material characteristics for the problem of bending of thin plates, J. Optim. Th. Appl. 42, 305-316.

[12] Saburi, T. and Nenno, S. (1981) The shape memory effect and related phenomena, in H.I. Aaronson et al. (eds.), Proc. Int. Conf. on Solid-Solid Phase Transformations, The Metall. Soc. AIME, New York, pp. 1455-1479.

[13] Shu, Y. C., PhD. Thesis, California Institute of Technology, in preparation.

[14] Shu, Y. C., and Bhattacharya, K. (1997) The effect of texture on the shapememory effect in polycrystals, in preparation.

[15] Šveràk, V. (1992) Rank-one convexity does not imply quasiconvexity, Proc. Royal Soc. Edin. 120A , 185-189.

[16] Taylor, G.I. (1938) Plastic strain in metals, J. Inst. Metals 62, 307-324. 